\title{
MNMU-RA: Most Nearest Most Used Routing Algorithm for Greening the Wireless Sensor Networks
}

\author{
Hafiz Bilal Khalil, Syed Jawad Hussain Zaidi \\ School of Electrical Engineering \& Computer Sciences, National University of Sciences and Technology, Islamabad, Pakistan \\ Email: \{10mseetkhalil, 10mseejzaidi\}@seecs.edu.pk
}

Received February 22, 2012; revised March 22, 2012; accepted April 10, 2012

\begin{abstract}
Wireless sensors are widely deployed in military and other organizations that significantly depend upon the sensed information in any emergency situation. One of the main designs issues of the wireless sensor network (WSN) is the conservation of energy which is directly proportional to the life of the networks. We propose most nearest most used routing algorithm (MNMU-RA) for ad-hoc WSNs which vitally plays an important role in energy conservation. We find the best location of MNMU node for energy harvesting by apply our algorithm. Our method involves the least number of nodes in transmission of data and set large number of nodes to sleep in idle mode. Based on simulation result we shows the significant improvement in energy saving and enhance the life of the network.
\end{abstract}

Keywords: Energy Efficiency; Wireless Sensor Networks; Routing

\section{Introduction}

The growth in wireless sensor networks and its applications dramatically increased in last decade. Wireless sensor nodes are widely used in military surveillance, intelligence and targeting in war operations. Energy available at each sensor for sensing and communications is limited because of the cost constraints and smaller size, which affects the sensor application and network lifetime. The purpose of green networking is to overcome the carbon foot print, reduce the energy consumption and energy losses. Energy efficiency is an important issue to enhance the life time of the network. To achieve the green networking every component of the network is integrated with energy efficient protocols, e.g., energy-aware routing on network layer, energy-saving mode on MAC layer, etc. One of the most important components of the sensor node is the power source. In sensor networks generally there are three modes of power consumption: sensing, data processing, and communication. Compared to sensing and data processing, much more energy is required for data communication in a typical sensor node [1]. These are also categorized as sleep (idle) and wakeup (transmission) mode.

In ad-hoc WSNs (Wireless Sensor Networks) always the nodes are cooperative, they sense and transmit their own data and also act as router to route the sensed information of other nodes towards the data center or gateway node which is connected to the internet. Most of the nodes consumed their power resource while transmitting the data of neighboring nodes. The scope of this paper is to minimize the power consumption in transmitting or routing process and set large number of nodes into sleep mode. The remaining sections of this paper organized as follows. Section 2 explains related work and current energy efficient techniques for sensor networks. Section 3 introduces some problems and research issues in current work. Section 4 describes overview of network model, our proposed algorithm and proposed solution respectively. In Section 5 experiment, Results and comparisons are given.

\section{Related Work}

Energy efficiency is already achieved by many approaches. These approaches include energy aware protocol development and hardware optimizations, such as sleeping schedules to keep electronics inactive most of the time, dynamic optimization of voltage, and clock rate. In [2] Smart Dust motes are designed that are not more than a few cubic millimeters. They can float in the air, keep sensing and transmitting for hours or days. In [3] authors described the $\mu$ AMPS wireless sensor node, it is hardware based solution in which they simultaneously consider the features of the microprocessors and transceivers to reduce the power consumption of the each wireless sensor node in network. Routing algorithms also play an important role to reduce the energy consumption during the routing of data. A lot of work is done in MAC layer and Mac protocols; MAC protocol for wireless sensor 
networks is not like the traditional wireless MACs such as IEEE 802.11. One of the most important goals is energy conservation, fairness and latency is less important [4].

SMAC/AL (Sensor MAC with Adaptive Listening) is a famous MAC protocol for WSNs proposed by Ye et al. $[5,6]$. Main purpose of SMAC/AL is to reduce energy consumption. But in SMAC/AL without considering the distance among the nodes, all nodes unnecessarily consume the energy by transmitting information with constant power level. An energy efficient MAC protocol with adaptive transmit power scheme named ATPM (Adaptive Transmit Power MAC) is proposed in [7]. By measuring the received power ATPM can calculate the distance between the sender and the receiver, and then adaptively choose the suitable transmit power level according to the propagation model and distance. The ATMP can not only conserve the energy source, but also decrease the collision probability. A Novel Clustering Algorithm for Energy Efficiency in Wireless Sensor Networks (ANCAEE) has been proposed [8]. It minimizes energy utilization during data transmission and energy consumptions are distributed uniformly among all nodes. Each cluster contains cluster head, each node send its data to cluster head with single hop transmission. And cluster transmits the combined data to the base station with multi hope transmission. This approach reduces energy consumption of nodes within the cluster.

\section{Problem Statement}

Sensor nodes which are one hope away or closest to the gateway node always consume their power more quickly than others because they have to transmit the data of other nodes in addition to transmission of their own sensed information. In [9] a solution was proposed for such type of scenario by implementing the multiple base stations and periodically changing their positions. But the problem is that if every time the most far away sensor transmits its data then major part of overall network energy will be consumed. Another solution for prolong the sensor network lifetime is to divide sensors nodes into disjoint sets, such that all the targets completely covered by every set [3]. Authors consider that within an active sensor's operational range a target is covered. These disjoint sets are activated in round robin fashion, such that at a time only one set is active. Sensors are into the active state in an active set and all other sensors are in a lowenergy sleep state. According to this method almost half of the sensor remains active and remaining half goes to sleep mode which reduce energy down to $50 \%$. To make it more efficient and conserve the larger amount of energy we proposed an algorithm named as MNMU-RA (Most nearest most used routing algorithm). That algorithm finds the efficient placement of active sensor nodes and set other nodes into sleep mode. An issue is also resolved by our algorithm, reducing the number of multiple base stations by finding out the best location for the base station without changing its location periodically.

\section{Synopsis of Our Network Model}

In this paper we deal with the issue of energy efficiency in wireless sensor networks for surveillance of a set of targets with known locality. Scenario of the network is chosen for armed forces purposes like surveillance of the boarder, battle fields and no go areas to acquire the information about enemies and their locations without taking the risk for human personal. We consider that a large number of sensors are distributed randomly in close proximity for monitoring and send the monitored information to a gateway node. All nodes are static and makes ad-hoc wireless sensor network. Every sensor nodes must monitor the area all the time in its operational range and each sensor has fixed transmission range. In network model we assume that each sensor has unique pre configured Id and Global/proactive routing algorithms are used. Main advantage of proactive algorithm is not route latency but drawback is the high maintenance overhead when many of the routes are never used. Proactive routing is appropriate for networks with: Small size, low mobility and high communication rates. We proposed an algorithm called as most nearest most used routing algorithm for this purpose. By using MNMU-RA we can find the perfect location of node for energy harvesting which also reduce the overall energy consumption and cost.

\subsection{Most Nearest Most Used Routing Algorithm}

Run shortest path routing algorithm or link state routing to find the shortest path for each node in the wireless sensor network. Calculate all the possible shortest paths for each node. Then find the MNMU node (Figure 1).

- A node which is most nearest to the gateway node.

- Select a node which is used in maximum number of shortest paths.

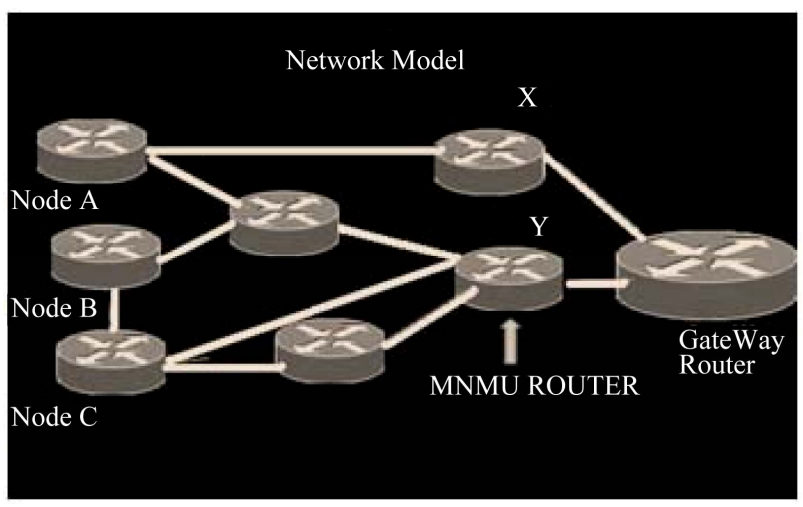

Figure 1. Location of selected MNMU node. 
In above network model we assumed that sensed information is equally probable for all the nodes. Then we calculate the shortest path for the nodes A, B and C. Then we find out the nodes which are most nearest to the gate way node. In above network model there are only two nodes $\mathrm{X}$ and $\mathrm{Y}$ which are closer to the gateway node. Then for selection we give the preference to the node which is most used in shortest paths. In above model $\mathrm{Y}$ is node which is most used in all shortest paths. If nodes A, $\mathrm{B}$ and $\mathrm{C}$ transmit their data the entire time node $\mathrm{Y}$ will be included in their path. Then every node keeps its routes information towards the node $\mathrm{Y}$ for future communications. Flow chart of our algorithm is given in Figure 2.

\subsection{Proposed Solution}

We used our algorithm to find most nearest most used node in a network, that node should be active all the time while other sensors remain in sleep mode and keep sensing. As we use proactive routing so each sensor knows its path towards the MNMU node. If a node has to send its information before sending it will wake up the nodes along his route. When MNMU nodes receive the information it will forward the data to the gateway and sets all the nodes into sleep mode. The critical issue in this solution is that if a node (MNMU node) remains active all the time then its energy source will be empty soon. We resolve this issue by using the energy harvesting concept at MNMU node [1]. We can also use secondary battery

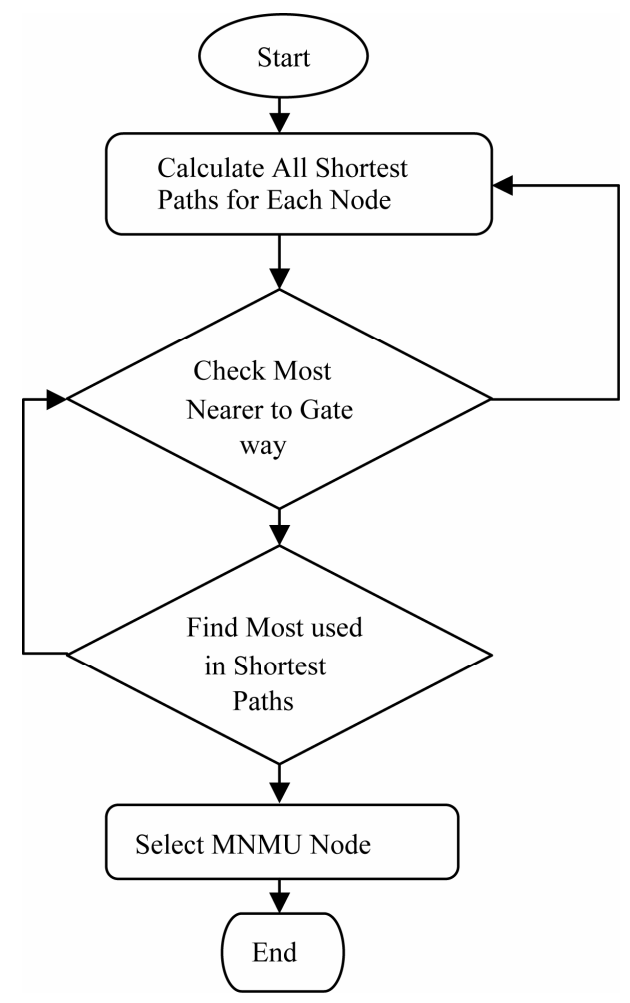

Figure 2. MNMU routing algorithm flow chart. which is rechargeable and coupled with photovoltaic cell [10]. If all the nodes can generate energy from light, vibration, heat etc $[11,12]$ it will increase the system cost. We don't need to replace all the nodes with secondary sources. By replacing only one node (MNMU node) resolves the issue and slightly increases the cost of the overall system. But effectively prolong the life time of sensor network. A solution given by Gandham et al. [9] can be more energy efficient if we implement our proposed algorithm with every new location of mobile base station. Split the network in equal parts and periodically change the position of base station in each part. Base station can be easily implemented at the place of MNMU node in each part of the network instead of replacing it outside the network. MNMU node will reduce the multi hop and number of transmission which directly reduce the energy consumption.

\section{Experiment}

We done the experiment by implementing our proposed algorithm in a network and calculate the amount of energy utilization using MATLAB. Then implement the concept of disjoint set and analyze the values at same network. For simulation 20 nodes containing one gateway node are distributed randomly in 30 meter square area. We consider the features of MICA2 mote platform. It is third generation mote specifically built for WSNs [4]. MICA2 have selectable transmission power range which offers adjustable communication ranges, selected transmission range for each node is 10 meters. The packet length is fixed at 200 bits. MICA2 usually operated with $3 \mathrm{~V}$ battery and other features mentioned in Table $\mathbf{1}$.

We divided our analysis in three parts; first we calculate the power consumption using disjoint sets methods [3], then we apply our algorithm and calculate \& compare power consumption. Same network and topology taken in which each node remains active all the time and no energy saving protocol and technique is implemented. Energy calculated during the 20 rounds, all nodes are active in first five rounds in which they sense and transmit the data. After ten rounds there is no activity and nodes go to sleeping mode according to implemented

Table 1. Features of MICA2 motes platform [12,13].

\begin{tabular}{cc}
\hline Operation/Features & Unit \\
\hline Listening & $8 \mathrm{~mA}$ \\
Receiving & $10 \mathrm{~mA}$ \\
Transmission & $17 \mathrm{~mA}$ \\
Sleep & $19 \mu \mathrm{A}$ \\
Radio Frequency & $900 \mathrm{MHz}$ \\
CPU 8 bit & Atmel at $8 \mathrm{MHz}$ \\
Bandwidth & $40 \mathrm{Kbps}$ \\
\hline
\end{tabular}


methodology. Calculated results are given in Figures 3 and 4.

\section{Simulation Results}

Figure 3 shows the result comparison of energy consumption in different modes; sensing, Transmission and sleeping of network. In Figure 3(a) set of all the active nodes shown by blue line are transmitting the data without applying any energy saving protocol. During the transmission if all nodes are active they will keep transmitting the information to each other and maximum amount of energy is consumed. In disjoint system only active set take part in transmission and inactive nodes

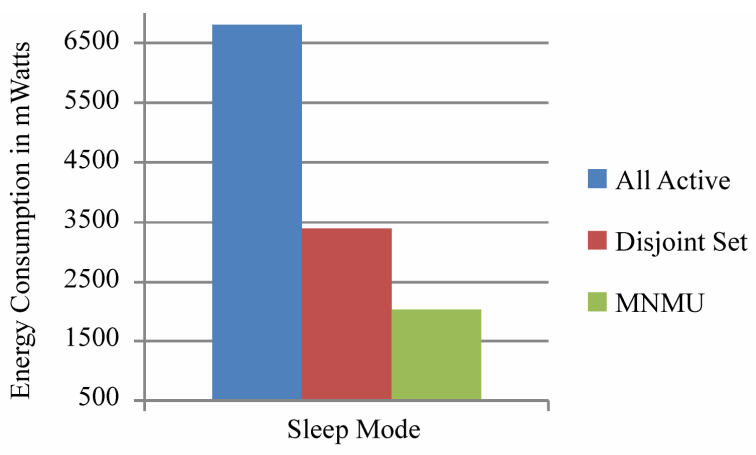

(a)

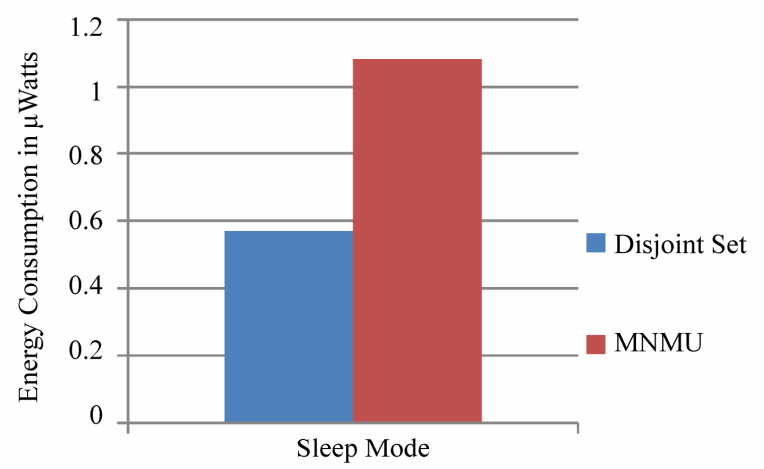

(b)

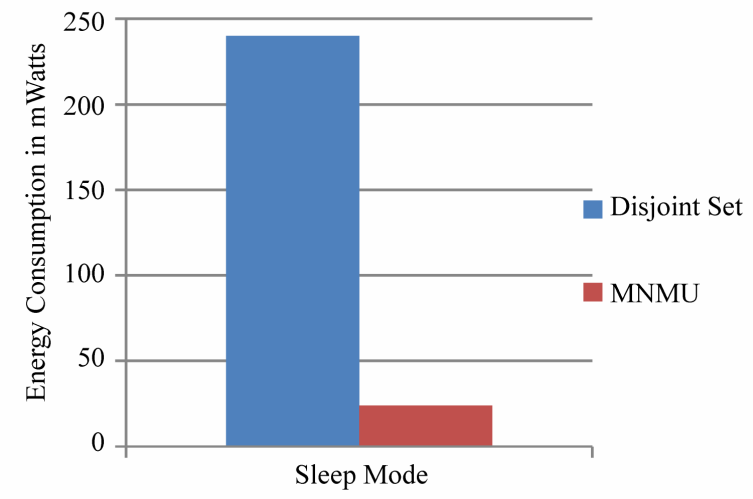

(c)

Figure 3. Power consumptions in different modes. (a) Transmission mode; (b) Power consume by sleeping nodes; (c) Power consume by active nodes in sleep mode.

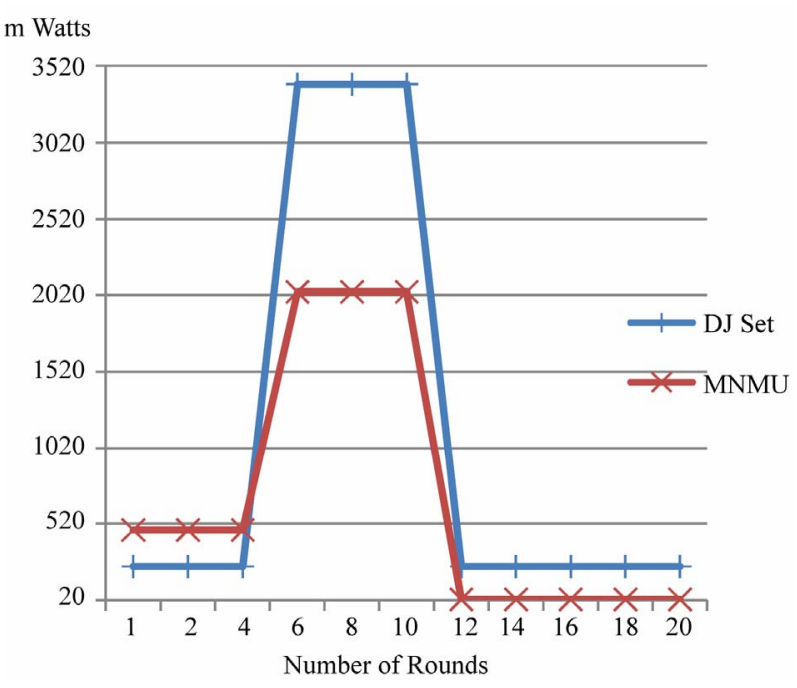

Figure 4. Result and comparison of energy consumption in different modes.

remain inactive during the transmission of active set. Our proposed algorithm gives lowest amount of energy consumption because only the MNMU node and less number of nodes take part in transmission. Energy consumed by inactive nodes in sleeping modes is shown in Figure 3(b). Energy consumption of sleeping nodes is in $\mu$ watts. According to our algorithm 19 nodes set to sleep mode and only one MNMU node is active. While Figure 3(c) shows the separately calculated energy consumption by active nodes when there is no activity and network is in idle mode. Similarly in sleeping mode only MNMU node remains active and rest of the network sets to sleep mode.

Figure 4 shows the result of energy consumption of entire network in different rounds. In first 5 rounds we assume that there is no sensed information to send; all the nodes are active in listening mode and keep sensing. In 5 to 10 rounds nodes are transmitting their sensed information to the gateway. After round 10 there is no activity and nodes set to sleep mode in sleep mode only energy consumed by active nodes are calculated and energy consumed by sleeping nodes which is in $\mu$ watts is neglected. Our algorithm gives the minimum energy consumption during the transmission in which fewer numbers of nodes take part in routing and also in sleep mode by keeping only MNMU node active.

\section{Conclusion}

We presented the most nearest most used routing algorithm to reduce the energy utilization in wireless sensor networks. Using this algorithm we find the best location of energy harvested node in a network. Our algorithm involves least number of nodes during transmission and keeps one node active in sleep mode. That significantly reduces the energy consumption during the transmission 
and sleep mode when there is no activity. An open research issue is the heterogeneity of energy resources of the nodes that must be resolved after practical implementation in any network. In our solution there is uneven energy consumption due to the topology of the network and nature of data flow. But that uneven energy consumption is helpful to reduce the energy consumption of entire network

\section{Future Direction}

Desired goal in wireless networks is energy efficiency to maximize the network life. Our algorithm can be used to find the location of cluster header quickly in novel clustering algorithm for energy efficiency in wireless sensor networks [8]. Further we can implement coding techniques to reduce the number of transmissions at MNMU node. Energy consumes per bit or per packet transmission can be reduce. Number of packets can be transmitted as a single packet by applying x-or Operations which reduces the energy but may cause of slighter delay. To apply this technique sensor nodes must be smarter and have ability to do this quickly.

\section{REFERENCES}

[1] I. F. Akyildiz, T. Melodia and K. Chowdhury, "A Survey on Wireless Multimedia Sensor Networks," Computer Networks, Vol. 51, No. 4, 2007, pp. 921-960. doi:10.1016/j.comnet.2006.10.002

[2] J. M. Kahn, R. H. Katz and K. S. J. Pister, "Emerging Challenges: Mobile Networking for Smart Dust,” International Journal of Communication Networks, Vol. 2, No. 3, 2000, pp. 188-196.

[3] M. Cardei and D. Z. Du, "Improving Wireless Sensor Network Lifetime through Power Aware Organization," Wireless Networks, Vol. 11, No. 3, 2005, pp. 333-340. doi:10.1007/s11276-005-6615-6

[4] Q. Hu and Z. Z. Tang, "An Adaptive Transmit Power Scheme for Wireless Sensor Networks,” 3rd IEEE Inter- national Conference on Ubi-Media Computing, Jinhua, 5-7 July 2010, pp. 12-16.

[5] W. Ye, J. Heidemann and D. Estrin, “An Energy-Efficient MAC Protocol for Wireless Sensor Networks,” Proceedings of the IEEE INFOCOM, New York, 23-27 June 2002, pp. 1567-1576.

[6] W. Ye, J. Heidemann and D. Estrin, "Medium Access Control with Coordinated Adaptive Sleeping for Wireless Sensor Networks," IEEE/ACM Transactions on Networking, Vol. 12, No. 3, 2004, pp. 493-506. doi:10.1109/TNET.2004.828953

[7] Q. Hu and Z. Tang, “ATPM: An Energy Efficient MAC Protocol with Adaptive Transmit Power Scheme for Wireless Sensor Networks," Journal of Multimedia, Vol. 6, No. 2, 2011, pp. 122-128. doi:10.4304/jmm.6.2.122-128

[8] A. P. Abidoye and N. A. Azeez, "ANCAEE: A Novel Clustering Algorithm for Energy Efficiency in Wireless Sensor Networks,” Journal of Wireless Sensor Networks, Vol. 3, No. 9, 2011, pp. 307-312. doi:10.4236/wsn.2011.39032

[9] S. R. Gandham, M. Dawande, R. Prakash and S. Venkatesan, "Energy Efficient Schemes for Wireless Sensor Networks with Multiple Mobile Base Stations," Global Telecommunications Conference, San Francisco, 1-5 December 2003, pp. 377-381.

[10] M. A. M. Vieira, C. N. Coelho, D. C. Silva and J. M. Mata, "Survey on Wireless Sensor Network Devices," Proceedings of IEEE International Conference on Emerging Technologies and Factory Automation (ETFA'03), Lisbon, 16-19 September 2003, pp. 537-544.

[11] J. Paradiso and T. Starner, "Energy Scavenging for Mobile and Wireless Electronics," Pervasive Computing, Vol. 4, No. 1, 2005, pp. 18-27. doi:10.1109/MPRV.2005.9

[12] V. Gungor and G. Hancke, "Industrial Wireless Sensor Networks: Challenges, Design Principles, and Technical Approaches," IEEE Transactions on Industrial Electronics, Vol. 56, No. 10, 2009, pp. 4258-4265. doi:10.1109/TIE.2009.2015754

[13] CrossBow, Mica2 Data Sheet. http://www.xbow.com/Products/Product_pdf_files/MICA \%20data\%20sheet.pdf 